\title{
Atrophic Glossitis: Burning Agony of Nutritional Deficiency Anemia
}

\author{
${ }^{1}$ Neeti Swarup, ${ }^{2}$ Shreya Gupta, ${ }^{3}$ Chandrani Sagolsem, ${ }^{4}$ Zoya Chowdhary, ${ }^{5}$ Subhash Gupta, ${ }^{6}$ Nidhi Sinha
}

\begin{abstract}
Lingual atrophic condition is the loss of ordinary texture and appearance of the dorsal tongue, determined by papillary protrusion, which turns into a soft and smooth aspect. Atrophic glossitis (AG) is a lingual atrophic condition, characterized by loss of fungiform or filiform papilla from the dorsum of tongue. This is generally associated with pain, glossodynia, and burning sensation, glossopyrosis. It is associated with a variety of conditions, local and systemic. Atrophic glossitis is considered to be an important indicator for nutritional deficiency anemias. The study aims at a brief review of AG and its relation to nutritional deficiency anemia.
\end{abstract}

Keywords: Atrophic glossitis, Epithelial atrophy, Nutritional deficiency anemia.

How to cite this article: Swarup N, Gupta S, Sagolsem C, Chowdhary Z, Gupta S, Sinha N. Atrophic Glossitis: Burning Agony of Nutritional Deficiency Anemia. World J Anemia 2017;1(2):48-50.

Source of support: Nil

Conflict of interest: None

$1,3,4$ Postgraduate Student, ${ }^{2,6}$ Senior Lecturer, ${ }^{5}$ Assistant
Professor

${ }^{1}$ Department of Oral Pathology and Microbiology, Teerthanker Mahaveer Dental College \& Research Centre, Moradabad Uttar Pradesh, India

${ }^{2}$ Department of Oral Pathology and Microbiology, Bhabha College of Dental Sciences, Bhopal, Madhya Pradesh India

${ }^{3}$ Department of Conservative Dentistry and Endodontics Teerthanker Mahaveer Dental College \& Research Centre Moradabad, Uttar Pradesh, India

${ }^{4}$ Department of Periodontology, Teerthanker Mahaveer Dental College \& Research Centre, Moradabad, Uttar Pradesh India

${ }^{5}$ Department of Ophthalmology, RKDF Medical College Hospital \& Research Center, Bhopal, Madhya Pradesh, India

${ }^{6}$ Department of Oral Pathology and Microbiology, New Horizon Dental College \& Research Institute, Bilaspur, Chhattisgarh India

Corresponding Author: Neeti Swarup, Postgraduate Student Department of Oral Pathology and Microbiology, Teerthanker Mahaveer Dental College \& Research Centre, Moradabad Uttar Pradesh, India, e-mail: drneetiswarupomfp@gmail. com

\section{INTRODUCTION}

Atrophic glossitis is also known as smooth tongue because of the smooth, glossy appearance with a red or pink background (Fig. 1). The smooth quality is caused by the atrophy of filiform papillae, described by Reamy et al. ${ }^{1}$ Partial or complete loss of fungiform and filiform papillae on the dorsal surface of tongue manifests as AG. It is a condition with multifactorial etiology, and can be a manifestation of underlying local or systemic condition. They may include nutritional deficiency, riboflavin, niacin, pyridoxine, vitamin B12 (pernicious anemia), folic acid, iron (iron deficiency anemia and Plummer-Vinson syndrome), protein-calorie malnutrition, infections, alcohol abuse, gastrointestinal diseases, and drug reactions. ${ }^{2}$ Histologically, AG is characterized by epithelial atrophy and varying degrees of chronic inflammation in the subepithelial connective tissue (Fig. 2). ${ }^{3}$

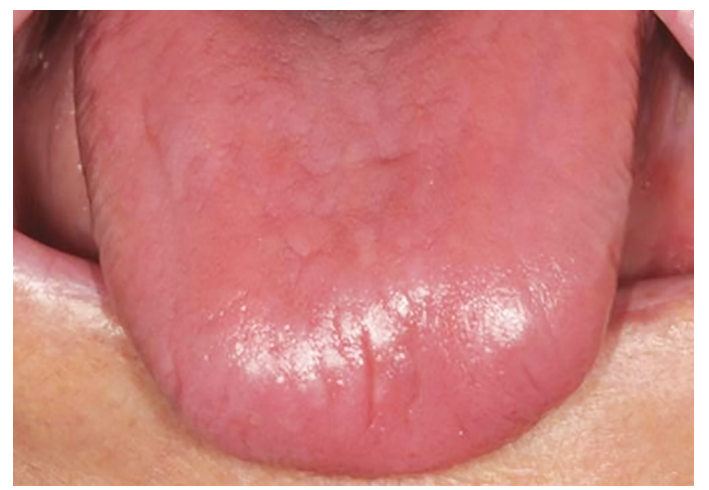

Fig. 1: Glossy appearance of tongue

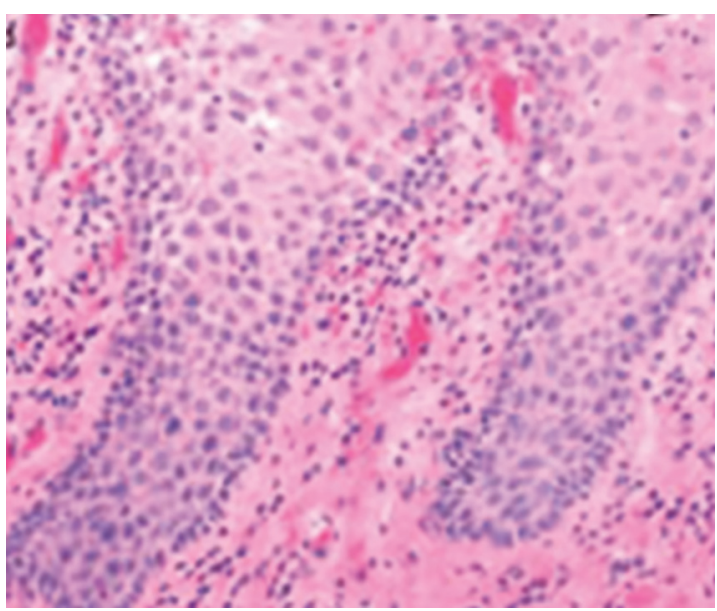

Fig. 2: Histopathology of atrophic glossitis 


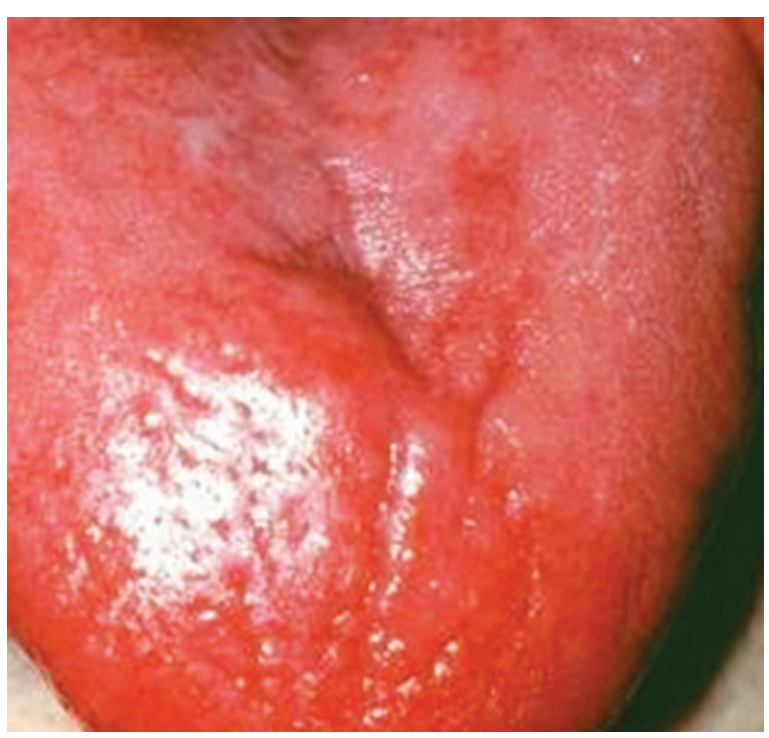

Fig. 3: Atrophic glossitis

\section{ANEMIAS MANIFESTING ATROPHIC GLOSSITIS}

\section{Normochromic Normocytic Anemia}

Associated with vitamin B2/riboflavin deficiency, normal plasma level being 1 to $19 \mu \mathrm{g} / \mathrm{L}$, normochromic normocytic anemia leads to magenta-colored glossitis. ${ }^{2,4}$ It is generally associated with deficiency of vitamin B6/pyridoxine, which further worsens the condition. ${ }^{5}$ Vitamin B2/riboflavin plays a major role in oxidation and reduction reactions. The deficiency of the same leads to epithelial atrophy, also associated with it is flattening of filiform papilla (Fig. 3). ${ }^{6}$

\section{Megaloblastic Anemia}

Associated with vitamin B12/cyanocobalamin and/ or B9/folic acid deficiency, normal vitamin B12 ranges from 200 to $900 \mathrm{pg} / \mathrm{mL}$. Normal vitamin B9 for adults ranges from 2 to $20 \mathrm{ng} / \mathrm{mL}{ }^{2,7,8}$ Macrocytic anemia may be normochromic/hypochromic. It is also characterized by hypersegmented neutrophils and plays a major role in the synthesis of deoxyribonucleic acid and ribonucleic acid, in the prevention of genetic alterations, and is required for epithelial maturation. Tongue appears as beefy/fiery red. ${ }^{2}$

\section{Pernicious Anemia}

This anemia is caused by vitamin B12 deficiency. Castle's intrinsic factor (CIF) is required for vitamin B12 absorption. Deficiency of CIF is responsible for this anemia. Other factors like vegan diet, celiac disease, tapeworm infections are also responsible for the same. The tongue appears as smooth red.
Atrophic glossitis associated with vitamin B12 and/ or vitamin B9 are also termed as bald tongue, Hunter glossitis, and Moeller glossitis.

\section{Iron Deficiency Anemia}

Iron deficiency anemia is morphologically characterized by microcytic hypochromic anemia. Iron plays a major role in hemoglobin formation. As hemoglobin is essential for transport of oxygen, iron deficiency affects this process significantly. ${ }^{2,9}$ Also iron is required for proper development and epithelial maturation, and it affects various epithelial structures. ${ }^{10}$

\section{DIAGNOSIS}

Atrophic glossitis is one of the lingual atrophic condition and needs to be differentiated from other lingual atrophic conditions, such as geographic tongue (benign migratory glossitis) and the median rhomboid glossitis. It is mostly associated with nutritional deficiencies. ${ }^{2}$

\section{MANAGEMENT}

Antibiotics, antifungal medications, or other antimicrobials may be prescribed if the glossitis is due to an infection. Dietary changes and supplements are used to treat anemia and nutritional deficiencies. Avoid irritants (such as hot or spicy foods, alcohol, and tobacco) to reduce any tongue discomfort. Treatment usually aims at reducing inflammation by corticosteroids. Other preventive measures include maintaining proper oral hygiene, irritants like hot, spicy foods, and alcohol should also be avoided to recover from this disorder and minimize discomforts. Suffering individuals must give up smoking.

\section{DISCUSSION}

Tongue can be considered as a mirror of oral and/or systemic health. An oral medicine expert can be the first to observe the lingual status, and should be familiar with the different diagnoses of these conditions, e.g., being able to link lingual abnormalities with specific etiologic causes. Recognition of alterations relative to tongue's morphology, in the presence of a negative anamnesis, should lead to more accurate investigations, in order to ascertain if they are manifestations of an underlying systemic condition.

Amid tongue diseases, AG has the utmost complex differential diagnosis, since it is allied with several conditions. Atrophy of the filiform papillae is not directly attributable to mechanical damages of the mucosa, and occurs in systemic or local conditions. Though apparently simple, sometimes the recognition of a "real AG" can be very challenging. 


\section{CONCLUSION}

Primary recognition of signs and symptoms relative to nutritional deficiency anemia through careful analysis of oral anatomical structures appears vital in the perspective of achieving a better prognosis. Atrophic anomalies are linked to numerous conditions, both local and systemic, that clinicians ought to recognize and identify. Knowledge of oral diseases and their etiology is important for these reasons, which necessitates an unceasing professional education along with interaction with other medical specialists when needed.

\section{REFERENCES}

1. Reamy BV, Derby R, Bunt CW. Common tongue conditions in primary care. Am Fam Physician 2010 Mar;81(5):627-634.

2. Erriu M, Pili FM, Cadoni S, Garau V. Diagnosis of lingual atrophic conditions: associations with local and systemic factors. A descriptive review. Open Dent J 2016 Nov;10(1):619-635.

3. Sun A, Lin H, Wang Y, Chiang C. Significant association of deficiency of hemoglobin, iron and vitamin B12, high homocysteine level, and gastric parietal cell antibody positivity with atrophic glossitis. J Oral Pathol Med 2011 Jul;41(6):500-504.

4. Priemer, DS.; Staros, EB. Vitamin B2 (Riboflavin). New York (NY): Medscape; 2013. Available from: https://emedicine. medscape.com/article/2088605-overview.

5. Park KK, Brodell RT, Helms SE. Angular cheilitis, part 2: nutritional, systemic, and drug-related causes and treatment. Cutis 2011 Jul;88(1):27-32.

6. Wynder EL, Klein UE. The possible role of riboflavin deficiency in epithelial neoplasia. I. epithelial changes of mice in simple deficiency. Cancer 1965 Feb;18(2):167-180.

7. U.S. National Library of Medicine. Vitamin B12 level. Bethesda (MD): U.S. National Library of Medicine; 2017. Available from: https://medlineplus.gov/ency/article/003705.htm.

8. Ghadaban, R.; Almourani, R.; Staros, EB. Folate (Folic acid). New York (NY): Medscape; 2013. Available from: https:// emedicine.medscape.com/article/2085523-overview.

9. Raju V, Arora A, Saddu S. Atrophic glossitis; an indicator of iron deficiency anemia: report of three cases. Int J Dent Clin 2014;6(3):30-31.

10. Lu SY. Perception of iron deficiency from oral mucosa alterations that show a high prevalence of Candida infection. J Formos Med Assoc 2016 Aug;115(8):619-627. 